\title{
DESIGN OF A COMPRESSIVE DATA GATHERING SCHEME WITH AN ENERGY EFFICIENT RECONSTRUCTION
} ALGORITHM FOR WSN

\author{
Aman Jindal $^{1}$, Kanika Sharma ${ }^{2}$
}

Abstract-The principle objective of wireless sensor network is not only to perform the operation of data communication but also to increase the network lifetime by retaining the energy of nodes for the longer time. As sensor nodes are resource constraints to achieve this objective there is requirement of efficient optimization of network resources which can be achieved by doing efficient compression. Compressive Sensing is the best way to enhance he network lifetime as it prefers multi-hopping for communication and data aggregation to reduce the number of transmission to the base station. Compressive Sensing is one of the emerging techniques that serve compression and recovery for signal of interest. At $\mathrm{K}=10$, proposed method saves 250 number of measurements than random selection method. Moreover, at $K=150$, the conventional method needs almost 450 more measurements than the proposed scheme. The simulation results show that the proposed method support reduction in the number of measurements by $\mathbf{7 0 ~} \mathbf{8 0 \%}$ for wide range of sparsity level of high and low SNRs.

\section{Introduction}

WSN is a highly distributed and organized network that empowers applications for critical decision-making through collaborative computing, communications, and distributed sensing [1-3]. Over the past decade Wireless Sensor Networks (WSNs) is rapidly growing area because of fast advances in Micro electro-mechanical system (MEMS) technology, low power digital and analog circuitry, RF design, and wireless communication and sensor technology. WSN is a self-configured and infrastructure less wireless network that is utilized for performing monitoring, tracking and detecting operations in real time environmental [4-5].

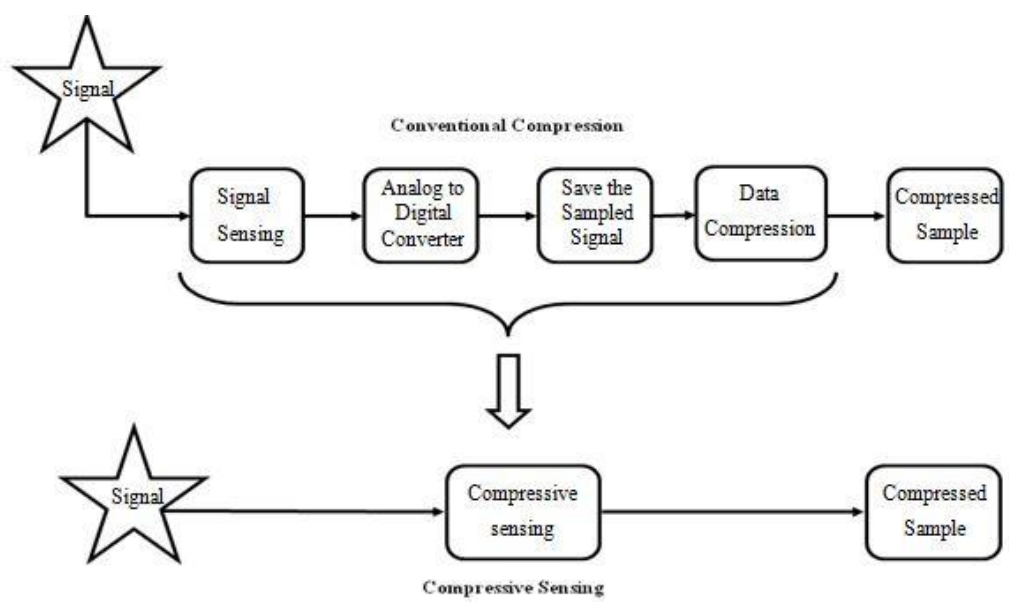

Fig.1 Fundamental idea behind CS

Shannon's Nyquist theorem [6] states that when uniformly signal sampling in order not to lose the information we must sample the signal by two times or more times faster than its bandwidth. Various applications, such as digital image and video cameras, to transmit or store the information samples must be compressed. This approach is followed by several compression schemes, for example, the JPEG format for images, MP3 for audio or video data.

It is inefficient to use the conventional compression because signal processing stages requires it unnecessary. As shown in Fig.1, the CS provides a new concept of compress the data without processing through the various stages of conventional

\footnotetext{
${ }^{1}$ M.E. Student, Department of ECE, NITTTR, Chandigarh

2 Assistant Professor, Department of ECE, NITTTR, Chandigarh
} 
compression [7]. Compressive sensing additionally performs the reconstruction of original signal from the compressed signal with minimum error [8].

\section{Related Work}

Mohamed Younis et al., in [9] have proposed a algorithm that helps to overcome the energy inefficiencies of the system that occur on the account of long haul communication which occurs when the link has to be made at a distant location. The lifetime of network gets reduced in such cases. The proposed algorithm avoids this by selecting proper gateway which is less energy constrained that is assigned as $\mathrm{CH}$. This algorithm helps balancing load in network. Ebrahimi Dariush.et.al.in [10] have presented a decentralized method for the compressive data gathering problem (DCDG). Method allows each sensor node to locally make a decision in constructing and maintaining the forwarding trees and has minimal complexity and overhead with outstanding performance. Sai Ji.et.al.in [11] have investigated how Energy consumption affecting the lifetime of wireless sensor networks (WSNs) in structural health monitoring (SHM). A huge original acquisition data was transmitted between nodes which occupy a large amount of communication bandwidth, and even lead to paralysis of WSNs. Yu Tang.et.al.in [12] Algorithm has been developed in order to recover the outlying sensor readings. Simulation result shows that algorithm provides less Average relative error and better detection ratio of outlying sensor readings as compared to wavelet and entropy algorithms. D.Brunelli.et.al.in [13] have proposed a new approach to simultaneous sensing and compressing that is highly promising for fully distributed compression in wireless sensor networks (WSNs). In this paper the two most prominent frameworks on sparsity and compressibility of multidimensional signals and signal ensembles, distributed compressed sensing (DCS) and Kronecker compressive sensing (KCS), are investigated. Qing Ling.et.al.in [14] have developed an optimal decentralized algorithm for sparse signal recovery and demonstrates its application in monitoring localized phenomena using energy-constrained large-scale wireless sensor networks. Liu Xiang.et.al.in [15] have investigated the application of CS to data collection in wireless sensor networks, and aim at minimizing the network energy consumption through joint routing and compressed aggregation. Author first characterize the optimal solution to this optimization problem, and then prove its NPcompleteness.

\section{Problem Definition}

The Proposed Work enhances the network lifetime and reduces the Mean Square Error of the network by using the efficient compressive sensing technique with the reconstruction algorithm based on the spatial correlation. There are three phases: Data gathering phase and Reconstruction Phase. Data Gathering phase is further divided in Set up phase for data collection at cluster head and Compression phase for the generation of sparse signal.

\section{Proposed Work}

a) Initialization of Parameters

There are some Network parameters are shown in Table 1. This table gives the value of different parameters like Sampling frequency, number of nodes, number of spikes of original signal etc.

Table 1 Network Parameters [19]

\begin{tabular}{|l|l|l|}
\hline Parameter & Description & Value \\
\hline $\mathrm{F}_{\mathrm{s}}$ & Sampling Frequency & $100 \mathrm{MHz}$ \\
\hline $\mathrm{N}$ & Number of Sensor Nodes & 1000 \\
\hline $\mathrm{k}$ & Number of spikes of original signal & 10 \\
\hline $\mathrm{c}$ & constant & 2.5 \\
\hline $\mathrm{M}$ & Number of measurements & 80,220 \\
\hline $\mathrm{x}$ & Original signal & random \\
\hline
\end{tabular}

\section{b) Generation of Random Original Signal}

First of all, the original signal $\mathbf{x}$ should be created to simulate the signal exist in the nature world. Base on the theory, this signal should be sparse in some domain. As it is created in MATLAB, definitely, it is a digital signal. The data length N of the original signal $\mathbf{x}$ will be set to 1000 and the sample frequency will be $100 \mathrm{MHz}$. The sine wave will be constructed as equation (1) [19]

$$
\begin{aligned}
& \mathrm{X}=\mathrm{B}(\mathrm{i}) \sin (2 \mathrm{f}(\mathrm{i}) \mathrm{t}+\theta(\mathrm{i})) \\
& \text { Where } \mathbf{X}=\text { the original signal, } \\
& \qquad \begin{array}{l}
\mathbf{B}(\mathbf{i})=1,2 \ldots, \mathrm{N} \\
\mathbf{f}(\mathbf{i})=\text { the amplitude, } \\
\mathbf{t}=\text { the time } \\
\boldsymbol{\theta}(\mathbf{i})=\text { the phase of the signal. }
\end{array}
\end{aligned}
$$

\section{c) Apply Spatial correlation function}

The correlation function can be used for the following two purposes:

- To detect non-randomness in data. 
- $\quad$ To identify an appropriate time series model if the data are random.

The function i.e. used on signal in eq. (8) is shown in eq.(2) [19]

$\mathrm{G}_{\mathrm{i}}=\left(\mathrm{W}_{\mathrm{i}}\right) \operatorname{corr}\left(\mathrm{W}_{\mathrm{i}}\right) ; \quad \mathrm{i}=1,2 \ldots, \mathrm{P}$

Where $\mathbf{G}=$ signal after applying autocorrelation function,

$\mathbf{w}=$ original signal

\section{d) Apply Fourier Transform}

Use the FFT to change the original signal $\mathbf{y}$ from time domain to frequency domain. The functions of FFT in MATLAB can be used directly to compute the fast discrete Fourier transform of signal $\mathbf{y}$ and rearranges the result of FFT by moving the zero frequency components to the middle of the spectrum.

The function i.e. used on signal in eq. (9) is shown in eq. (3) [19]

$\mathrm{Gf}=\mathrm{fftshift}(\mathrm{fft}(\mathrm{G}, \mathrm{N})) / \mathrm{N}$

Where $\mathbf{G f}=$ signal after applying Fourier transform

$\mathbf{G}=$ signal after applying spatial correlation function

$\mathbf{N}=$ No. of sensors nodes

\section{e) Generation of Measurement Matrix}

The digital signal $\mathbf{x}$ is presented as Nx1 vector in MATLAB. In order to collect the elements of this vector a measurement matrix should be created to sample this vector. As said in the theory Section 1.5.1.4, the random matrix can fulfill the conditions of CS with overwhelming probability for the measurement matrix. The matrix will be SxP dimensions matrix. The $M$ value is calculated through the equation (3).

The function i.e. used for generation of measurement matrix shown in eq.(4) [19]

$\mathrm{A}=\operatorname{randn}(\mathrm{M}, \mathrm{N})$

Where $\mathbf{A}=$ Generated measurement matrix

$\mathbf{M}=$ Number of measurements

$\mathbf{N}=$ No. of sensors nodes

\section{f) Reconstruction of Original Signal}

As mentioned before, in this case, convex optimal method will be used to get the sparsest solution to reconstruct the sparse signal $\mathrm{T}$. In MATLAB, CVX which is MATLAB-based modeling system for convex optimal programming will be used for convex optimal [19].

\section{g) Calculation of Mean Square error and Signal to Noise ratio}

The Parameters on the basis of which Performance of CDG is analyzed are MSE (Mean Square Error) and SNR (Signal to Noise Ratio).

\section{i. Mean Square Error}

Let $X$ and $\hat{X}$ be the true and the reconstructed reading, respectively. The Mean Square error (MSE) is defined to be the average of the ratio between the square of the difference of reconstruction reading and the true reading $v s$. the square of true reading [19]:

$M S E=\frac{\|X-\hat{x}\|_{2}}{\|x\|_{2}}$

Where $\mathbf{X}=$ the true original signal,

$$
\widehat{\boldsymbol{X}}_{=} \text {the reconstructed signal }
$$

\section{ii. Signal to Noise Ratio}

Signal to Noise Ratio is the ratio between signal power and the thermal noise power. Here we define two kinds of SNRs which are Total SNR (T-SNR) and Measured SNR (M-SNR).

T-SNR is the power ratio between uncompressed signal power and thermal noise power.[19]

$\mathrm{T}-\mathrm{SNR}=E\left[\frac{\|z\|_{2}^{2}}{\left\|n_{T}\right\|_{2}^{2}}\right]$

M-SNR is the instantaneous power ratio between compressed signal and noise [19]

$\mathrm{M}-\mathrm{SNR}=E\left[\frac{\|\emptyset z\|_{2}^{2}}{\left\|n_{M}\right\|_{2}^{2}}\right]$

Where, $\mathrm{z}=$ uncompressed signal, 


$$
\begin{aligned}
& \emptyset z=\text { compressed signal }, \\
& \mathrm{n}=\text { noise }
\end{aligned}
$$

h) Mathematical Model for Compressive Reconstruction Scheme

i. Autocorrelation function on original signal

Let's say autocorrelation function $\mathbf{R}$ applied to the Original random signal $\mathbf{x}(\mathrm{Nx} 1)$ and it will give the signal let's say $\mathbf{y}$ (Nx1) [15]

$$
\mathbf{R}=\sum_{\mathbf{n}=0}^{\infty} \mathbf{x}[\mathbf{n}] \mathbf{x}[\mathbf{n}-\mathbf{j}]
$$

\section{ii. Sparse representation of the auto correlated signal}

Signal $\mathbf{y}(\mathrm{Nx} 1)$ will have a sparse expression on the represent basis $\mathfrak{£}(\mathrm{N} x \mathrm{~N}), \mathrm{N}$ is the data length of signal $\mathbf{y}$ [18]:

$\mathbf{Y}=\mathfrak{£} \mathbf{k}$

Where $\mathbf{y}=$ auto correlated signal

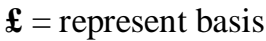

$\mathbf{k}=$ sparse represent of original signal

iii. Acquire the measurement value by measurement matrix

Use the measurement matrix $\boldsymbol{\Phi}(\mathbf{M x ~ N})$ to acquire the measurement value $\mathbf{A}, \mathbf{M}$ is the measurement number [19]:

$\mathbf{A}=\mathbf{y} \boldsymbol{\epsilon}=\boldsymbol{€} \mathfrak{£} \mathbf{k}$

Where $€=$ measurement matrix

\section{iv. Reconstruction of signal}

Choose an adaptive algorithm to reconstruct $\mathbf{k}$ depending on the known $£$, $\boldsymbol{€}$ and $\mathbf{A}$.

Using the inverse matrix of $£$ to reconstruct the original signal $\mathbf{y}^{*}[19]$.

$$
\mathbf{Y}^{*}=\mathbf{f}^{-1} \mathbf{T}^{*}
$$

Where $\mathbf{Y}^{*}=$ reconstructed original signal

$$
\mathbf{k}^{*}=\text { reconstructed sparse represent of original signal }
$$

\section{Results and Discussions}

Improved Compressive Data gathering and reconstruction scheme is implemented and tested in this chapter. Proposed algorithm increases the network lifetime by reducing the number of measurements and improves the Signal to Noise Ratio and reducing the Mean Square Error and hence making the network more energy efficient. Rest of this chapter consists of the simulation settings, MATLAB simulation results and results validation.

\subsection{Simulation Settings}

For the simulation MATLAB software is used. It is a tool that can simulate and test any simple mathematical model. Here in proposed work 1000 homogenous sensor nodes are deployed randomly in the square field of $100 * 100$ meter square. These sensor nodes remain fix throughout the network operation of communication. All the simulation parameters are given in Table3.

Table 3 Simulation Parameters

\begin{tabular}{|l|l|}
\hline Parameter & Value \\
\hline Shape of the network & Square \\
\hline No. of Sensor nodes & 1000 \\
\hline No. Sparse Signals & 10 \\
\hline Transmitter/receiver electronic circuit & $\mathrm{E}_{\mathrm{elc}}=\mathrm{E}_{\mathrm{tx}}=\mathrm{E}_{\mathrm{rx}}=50 \mathrm{~nJ} / \mathrm{bit}$ \\
\hline Data aggregation energy & $\mathrm{EDA}=5 \mathrm{~nJ} / \mathrm{bit} / \mathrm{Signal}$ \\
\hline Transmit amplifier(if d to BS $<\mathrm{d} 0)$ & Efs $=10 \mathrm{pJ} / \mathrm{bit} / \mathrm{m}^{2}$ \\
\hline Transmit amplifier(if d to BS $>\mathrm{d} 0)$ & Emp $=0.0013 \mathrm{pJ} / \mathrm{bit} / \mathrm{m}^{4}$ \\
\hline Fs & $100 \mathrm{MHz}$ \\
\hline C & 2.5 \\
\hline Original Signal & Random \\
\hline
\end{tabular}

a) Generation of random original signal

Random signals are random variables which evolve, often with time (e.g. audio noise), but also with distance (e.g. intensity in an image of a random texture), or sometimes another parameter. For the moment we shall assume that random signals are sampled at regular intervals and that each signal is equivalent to a sequence of samples of a given random process, as shown in the Fig 3. 


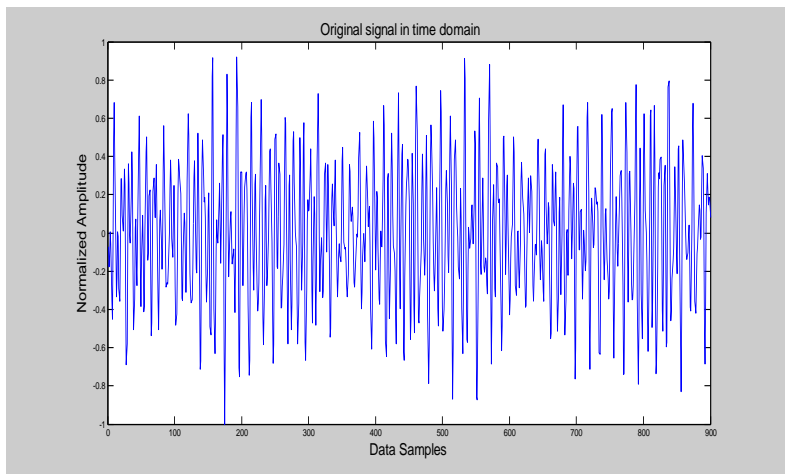

Fig. 3 Original signal in Time Domain

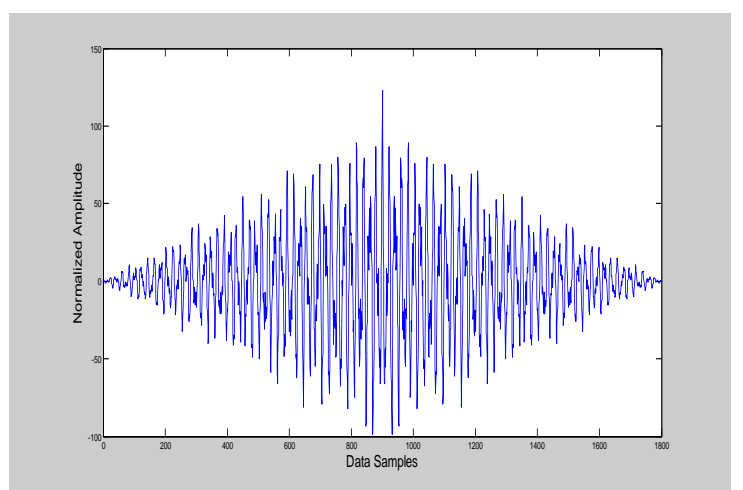

Fig 4 Signal after applying Spatial correlation function

\section{b) Applying Spatial correlation function on signal}

We consider a sensing architecture where $\mathrm{N}$ sensor nodes are spread over the field and collect data. The sensed data at ith sensor node as $\mathrm{x}_{\mathrm{i}}$ spatially correlated. An aggregrator receives these data sets from active sensor nodes. The Active and sleeping of the sensor nodes is depend upon the channel gain.

The proportion of good channels for given channel gain, $\mathrm{F}(\mathrm{p})$ as

$\mathrm{F}(\mathrm{p})=\frac{\text { The number of links that } p_{i} \geq p \text { for } \forall i}{\text { The number of total links }}$

Where $\mathrm{p}_{\mathrm{i}}$ is the channel gain of the ith sensor node. Thus for given $\mathrm{p}$ we can easily calculate the number measurements of which channel gain is larger than or equal to $\mathrm{p}$ as

$\mathrm{M}=\mathrm{F}(\mathrm{p}) \mathrm{X} \mathrm{N}$

Using this relationship, we can derive the channel gain threshold $\mathrm{p}_{\mathrm{thr}}$,

$\mathrm{p}_{\text {thr }}=\mathrm{F}^{-1}(\mathrm{M} / \mathrm{N})$

If the sensor nodes having channel gain greater or equal to threshold value then nodes goes in active node and transmits the data to the cluster head, otherwise it goes in sleep mode.

\section{c) Validation of Results}

\section{Measured SNR vs Number of target measurements}

In Fig. 6, examine the results of measured SNR versus the number of target measurements. The conventional random selection method shows the constant value of M-SNR of 50dB.On the other hand, the proposed selection method shows higher M-SNR than T-SNR and M-SNR decrease with increasing number of measurements.

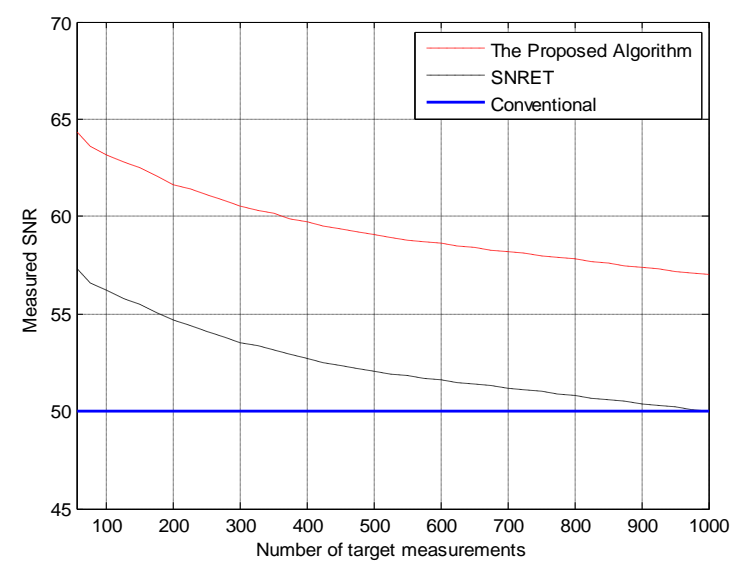

Fig 6 Measured SNR vs the number of measurement

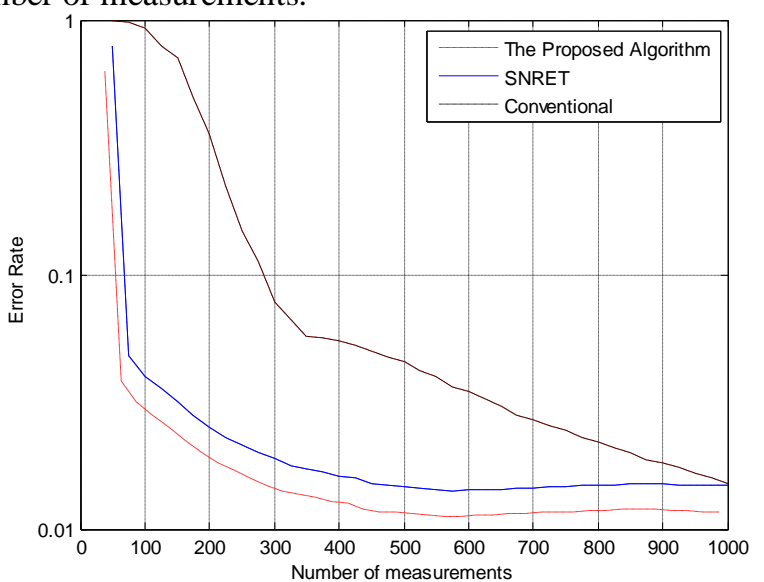

Fig 7 Error Rate vs the number of target measurements

\section{Error Rate vs Number of measurements}

In fig 7 shows the reconstruction error $\epsilon$ as a function of number of target measurements where T-SNR is $20 \mathrm{~dB}$. The size of target measurements varied from 50 to 1000 . It can be observed that proposed scheme outperforms the random selection method. The proposed scheme can save almost 500 measurements around $\epsilon=1.5 X 10^{-2}$. It is observe that when the number of measurements is about 500, it achieves performance of full sampling. This implies that with sufficient number of measurements for sparse signal recovery, measurements with low signal quality do not contribute to improving the detection 
performance any more. Thus, the proposed method with properly choosing threshold can prevent radio resources from being wasted while achieving the best possible performance.

\section{MSE ratio vs Number of target measurements}

To compare the efficiency of the proposed method with the random selection method more thoroughly, examined the MSE ratio $\frac{E_{\text {conventional }}}{E_{\text {proposed }}}$ between these two methods in Fig 8 . This results shows that the proposed method is relatively most

efficient for the number of measurements, 100.

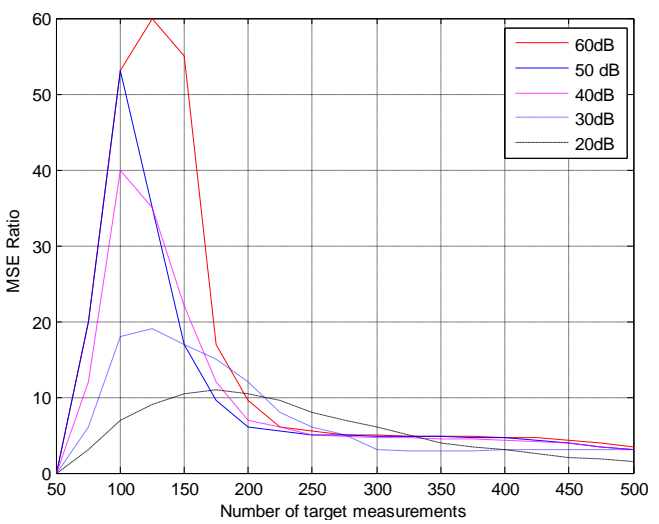

Fig 8 MSE ratio vs the number of measurements.

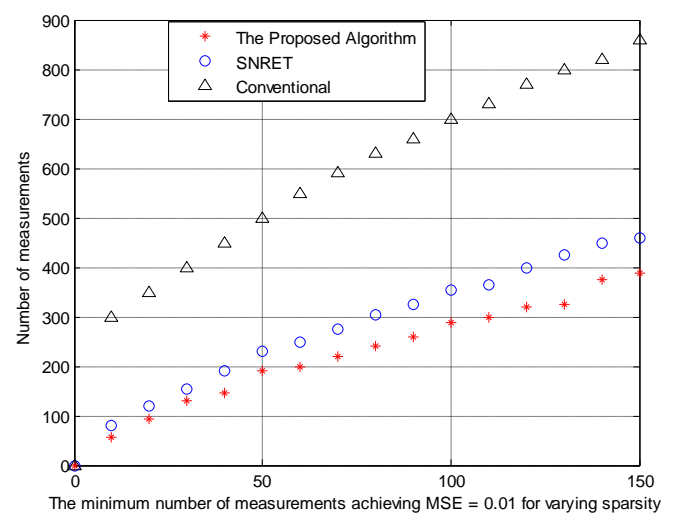

Fig 9 Number of measurements versus Sparsity

\section{Number of measurements versus Number of Sparsity signals.}

The relationship between the sparsity $\mathrm{K}$ and the number of measurements $\mathrm{M}$ for fixed T-SNR of 60dB in Fig 9. For each sparsity level, plotted the minimum number of measurements for which the normalized MSE is less than 0.01. This results show that the proposed method provides the consistent performance gain regardless of sparsity level. At $\mathrm{K}=10$ proposed method saves 250 number of measurements than random selection method. Moreover, at $\mathrm{K}=150$, the conventional method needs almost 450 more measurements than the proposed scheme.

\section{Conclusion}

In this paper, the proposed work is simulated in MATLAB environment and validated with existing SNR efficient transmission for compressive sensing based wireless sensor networks. The proposed scheme focuses on reducing reconstruction error by increasing the SNR of received measurements. To improve the SNR, we propose a signaling method with low overhead to achieve energy efficient transmission for a set of participating nodes. In the proposed work, it is observed that the proposed methods seemed to have error floor over the certain number of measurements. It may imply that some measurements with low quality may not contribute to improving signal recovery. More through theoretical approach may elucidate how detection algorithm process this information in recovery process, which possibly can give out the analytically minimum number of measurements to achieve the best possible performance in WSN having sparsity sparse observation. The proposed method provides the consistent performance gain regardless of sparsity level. At $\mathrm{K}=10$, proposed method saves 250 number of measurements than random selection method. Moreover, at $K=150$, the conventional method needs almost 450 more measurements than the proposed scheme. The simulation results show that the proposed method support reduction in the number of measurements by $70 \sim 80 \%$ for wide range of sparsity level of high and low SNRs.

\section{REFERENCES}

[1] Harvards volcanic WSNs, http://Fiji.eecs.harvard.edu/Volcano, 11 April, 2:20 pm, 2014.

[2] C. Cordeiro et al., "Ad Hoc and Sensor Networks: Theory and Applications", World Scientific Publishing, May 2006

[3] I. Akyildiz et al., "A survey on sensor networks" Communications Magazine, IEEE, Vol. 40, No. 8, pp-102-114, 2002.

[4] J. Yick et al., "Wireless sensor network survey", Computer Network, Vol. 52, No. 12, pp-2292-2330, 2008.

[5] F. Mieyeville et al., "Wireless sensor networks for active vibration control in automobile structures", Smart Materials and Structures, Vol. 21, No. 7, pp-075-099, 2012.

[6] K. Choi et al., "Compressed sensing based cone-beam computed tomography reconstruction with a first-order method", Med. Phys, Vol. 37, pp$5113-5125,2010$

[7] R. Baraniuk," Compressed sensing", IEEE Signal Processing, Vol. 24, 2007.

[8] D. Donoho.,"Compressed sensing", IEEE Trans. Inform. Theory, Vol. 52, No. 4, pp-1289-1306, Apr. 2006.

[9] Mohamed Younis and Gaurav Gupta, "Load-balanced clustering of wireless sensor networks" Communications, 2003.ICC'03.IEEE International Conference, Vol. 3, pp. 1848-1852, 2003.

[10] Xi Xu et al., "Power-efficient Nonuniform 2-D Fourier Analysis Using Compressive Sensing in WSN", IEEE Wireless Communications and Networking Conference, pp-1525-3511, April 2013.

[11] Mohammadreza Mahmudimanesh et al., "Robust Compressive data gathering in wireless sensor networks with linear topology "IEEE international Conference on Distributed Computing in Sensor System,pp.179-186,May 2014. 
[12] Ehrahimi Dariush et al., "A distributed method for Compressive Data gathering in wireless sensor network"IEEE Communiations letters,vol.15,No.4, PP.38-45,April 2014.

[13] Sai.Ji, Liping.Huang et al., "An improved Reconstruction method of Compressive data Recovery in Wireless Sensor Networks"International Journal of Security and Applications, Vol.8, No.1, pp.1-8,2014.

[14] Yu Tang, Bowu Zhang et al., "Robust Compressive Data Gathering in Wireless Sensor Networks".IEEE Transactions on Wireless Communications, Vol.12, No.6, June 2013.

[15] C.Caione et al.,"Compressive Sensing Optimization for Signal Ensembles in WSN”.IEEE Transactions on Industrial Informatics, Vol.10, No.1, pp.382-392, Feb 2014

[16] Qing Ling et al., "Decentralized Sparse Signal Recovery for Compressive Sleeping Wireless Sensor Networks". IEEE Transactions on Signal Processing, Vol. 58, No. 7, pp.3816-3827, July 2010.

[17] Liu Xiang et al., "Compressed data aggregation for energy efficient wireless sensor networks". 8th Annual IEEE Communications Society Conference on Sensor, Mesh and Ad Hoc Communications and Networks, pp, 46 - 54 ISSN, 2155-5486, June 2011.

[18] C.Caione et al., "Distributed compressive sampling for lifetime optimization in dense wireless sensor networks". IEEE Transactions on Industrial Informatics,Vol.8, No.1, pp.30-40, Oct 2011.

[19] Seunggye Hwang et al., "SNR efficient transmission for compressive sensing based wireless sensor networks", IEEE Transactions presented at IFIP WMNC, ISBN- 978-1-4673-5615-2, 2016 